\title{
Theory and Modeling of Lasing Modes in Vertical Cavity Surface Emitting Lasers
}

\author{
BENJAMIN KLEIN $^{\mathrm{a}, *}{ }^{*}$ LEONARD F. REGISTER $^{\mathrm{a}}$, KARL HESS $^{\mathrm{a}}$ and DENNIS DEPPE ${ }^{\mathrm{b}}$ \\ ${ }^{a}$ Beckman Institute and Coordinated Science Laboratory, University of Illinois at Urbana-Champaign, Urbana, Illinois 61801; \\ ${ }^{\mathrm{b}}$ Microelectronics Center, Department of Electrical and Computer Engineering, The University of Texas at \\ Austin, Austin, TX 78712-1084
}

\begin{abstract}
The problem of obtaining the lasing modes and corresponding threshold conditions for vertical cavity surface emitting lasers (VCSELs) is formulated as a frequency-dependent eigenvalue problem in required gain amplitudes and corresponding fields. Both index and gain guiding are treated on an equal footing. The complex gain eigenvalues define necessary but not sufficient conditions for lasing. The actual lasing frequencies and modes that the VCSEL can support are then determined by matching the gain necessary for the optical system in both magnitude and phase to the gain available from the laser's electronic system. Examples are provided.
\end{abstract}

Keywords: VCSELs, modes, lasing, modeling, gain eigenvalues

\section{INTRODUCTION}

In the simulation of edge emitting lasers, the optical field can usually be modeled simply by a predetermined set of Fox-Li quasimodes of the passive cavity [1]. The threshold condition can then be expressed as mode gain times mode lifetime equals unity. No laser previously has presented the challenge to optical simulation now presented by vertical cavity surface emitting lasers (VCSELs).

The challenge to VCSEL simulation is not just a much more complicated optical cavity geometry for analysis - although this problem alone is significant - but that quasi-mode analysis, itself, can no longer be relied upon. The mirrors are distributed and lateral confinement may be produced by a combination of index and gain guiding. (See, for example, Ref. [2].) Thus the VCSEL optical cavity boundaries, along with the passive cavity modes and conventional parameters such as the photon lifetime, are often poorly defined.

\section{THEORY}

At the threshold for lasing, the optical field will be self-supporting in the presence of the gain supplied

\footnotetext{
* Corresponding author.
} 
by the laser. Consider an open VCSEL optical cavity with tensor electric susceptibility $\stackrel{\leftrightarrow}{\chi}(\vec{r}, \omega)=$ $\stackrel{\leftrightarrow}{\chi}_{\text {cav }}(\vec{r}, \omega)+\overleftrightarrow{\chi}_{g}(\vec{r}, \omega)$ where $\stackrel{\leftrightarrow}{\chi}_{g}(\vec{r}, \omega)$ is the necessarily complex susceptibility representing the laser gain inside the active region provided under bias, and $\overleftrightarrow{\chi}_{\text {cav }}(\vec{r}, \omega)$ is the potentially complex susceptibility representing the rest of the VCSEL. Within the semiclassical approximation, lasing requires [3]

$$
\begin{aligned}
\vec{E}(\vec{r}, \omega)= & \int d \vec{r}^{\prime} \stackrel{\leftrightarrow}{G}_{\mathrm{cav}}\left(\vec{r}, \vec{r}^{\prime}, \omega\right) \\
& \cdot\left[j \omega \varepsilon_{o} \stackrel{\leftrightarrow}{\chi}_{g}\left(\vec{r}^{\prime}, \omega\right) \cdot \vec{E}\left(\vec{r}^{\prime}, \omega\right)\right]
\end{aligned}
$$

where $\vec{E}(\vec{r}, \omega)_{\leftrightarrow}$ is the electric component of the lasing field, $\vec{G}_{\text {cav }}\left(\vec{r}, \vec{r}^{\prime}, \omega\right)$ is the tensor Green's function for radiation from a current source within the open VCSEL cavity defined by $\stackrel{\leftrightarrow}{\text { cav }}_{\text {, }}$, and $j \omega \varepsilon_{o} \overleftrightarrow{\chi}_{g}\left(\vec{r}^{\prime}, \omega\right) \cdot \vec{E}\left(\vec{r}^{\prime}, \omega\right)$ acts as an equivalent current source.

If the spatial distribution of the gain is known to good approximation, then Eq. (1) reduces to an eigenvalue problem in the necessary gain amplitudes for lasing and the corresponding lasing fields. That is, if $\stackrel{\leftrightarrow}{\chi}_{g}(\vec{r}, \omega)=\kappa(\omega) \stackrel{\leftrightarrow}{\chi}_{g}^{(0)}(\vec{r})$ where $\overleftrightarrow{\chi}_{g}^{(0)}(\vec{r})$ is known, then Eq. (1) gives

$$
\frac{1}{\kappa(\omega)} \vec{E}(\vec{r}, \omega)=\hat{\mathcal{G}}(\omega) \vec{E}(\vec{r}, \omega)
$$

where $\hat{\mathcal{G}}(\omega)$ is an integral operator defined by

$$
\hat{\mathcal{G}} \vec{E}(\vec{r}) \equiv j \omega \varepsilon_{o} \int d \vec{r}^{\prime} \stackrel{\leftrightarrow}{G}_{\mathrm{cav}}\left(\vec{r}, \vec{r}^{\prime}\right) \cdot\left[\stackrel{\leftrightarrow}{\chi}_{g}^{(0)}\left(\vec{r}^{\prime}\right) \cdot \vec{E}\left(\vec{r}^{\prime}\right)\right] .
$$

Note that this eigenvalue problem need only be evaluated over regions for which $\stackrel{\leftrightarrow}{\chi}_{g}^{(0)}(\vec{r})$ is significant, that is, over the active region of the laser. The solutions to this eigenvalue problem will be complex gain eigenvalues and the corresponding fields as a continuous function of frequency, in contrast to the more familiar case of finding the discrete real frequency eigenvalues and modes of a closed passive cavity.

It is also possible to repartition the VCSEL susceptibility to take part of $\stackrel{\leftrightarrow}{\text { cav }}_{\text {out }}$ of the
Green's function and put it in Eq. (2) as a second source term. This may assist in the solution of problems for which the Green's function is not easily obtained. Extending the formulation in this way changes the ordinary eigenvalue problem of Eq. (2) into a generalized eigenvalue problem.

The solutions to Eq. (2) establish necessary but not sufficient conditions for the VCSEL to lase. The frequencies at which the VCSEL can actually lase are those for which the complex gain susceptibility necessary for the optical system can be matched to the complex gain susceptibility available from the laser electronic system in both magnitude and phase.

\section{EXAMPLE}

To illustrate the use of this formulation, lasing in the VCSEL cavity diagrammed in Figure 1 was considered [2]. This cavity allows for relatively easy analysis while allowing much of the essential physics of VCSELs to be modeled. $\chi_{\text {cav }}(\vec{r}, \omega)$ was taken as that of the planarly layered structure including the nominally lossy quantum well layer, but with no lateral confinment. Note, for simplicity, all susceptibilities were treated as scalars. The spatial distribution of the gain susceptibility was

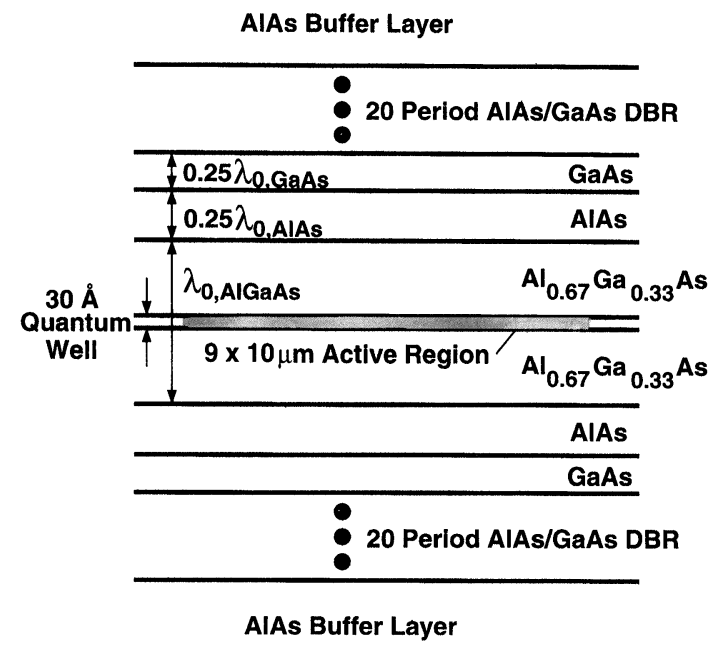

FIGURE 1 Model VCSEL cavity. $\lambda_{o}=980 \mathrm{~nm}$. 
approximated as uniform inside the active region and zero elsewhere. Thus for later convenience we set $\chi_{g}^{(0)}(\vec{r}) \equiv-j$ within the $9 \mu \mathrm{m} \times 10 \mu \mathrm{m} \times 3 \mathrm{~nm}$ quantum well active region, and $\chi_{g}^{(0)}(\vec{r}) \equiv 0$ elsewhere.

The Green's functions for such a planarly layered structure are well known [4], and only those with both source and field coordinates within the thin quantum well active layer were required. The Green's functions were obtained in Fourierspace where the only portion of the calculation that requires a computer is the calculation of the distributed-Bragg-reflector (DBR) reflectivities vs. frequency, incident angle and polarization, which may be performed using several equivalent methods $[5,6]$. Of course, calculation of the Green's function for more complex cavity structures could require computationally intensive numerical methods, while taking advantage of the cylindrical symmetry in many VCSELs could significantly reduce the computational load. However, while of obvious practical importance, how the Green's function is obtained or what coordinate system is used is of no conceptual importance in this formulation. Note that the potentially difficult problem of finding the Green's function would only need to be solved once for all bias conditions, as the Green's functions are independent of bias as long as effects such as thermal expansion of the cavity are ignored.

Once the Green's function was calculated, the gain eigenvalue problem of Eq. (4) was discretized and solved. Using a moment method, the field, approximated as constant over the width of the well, was expanded in rectangular testing functions with unknown complex coefficients in the $(x-y)$ plane of the well, i.e.

$$
\begin{array}{r}
\vec{E}(x, y)=\sum_{\vec{n}} \vec{E}(\vec{n}) \operatorname{rect}\left(\frac{x-n_{x} d_{x}}{d_{x}}\right) \\
\operatorname{rect}\left(\frac{y-n_{y} d_{y}}{d_{y}}\right),
\end{array}
$$

where $\operatorname{rect}\left[\left(\eta-\eta_{o}\right) / d\right]$ is a rectangular pulse of amplitude one and full width $d$ centered at $\eta_{o}$, and the discrete variable $\vec{n} \equiv\left(n_{x}, n_{y}\right)$ labels the grid sites. (For notational convenience, the various dependencies on frequency are no longer indicated explicitly.) Then the inner products of both sides of Eq. (4) were taken with the same rectangular testing functions (Galerkin's method [7]). The result was a finite set of linear equations for the unknown field coefficients $\vec{E}(\vec{n})$ of the same form as Eq. (2),

$$
\sum_{\vec{n}^{\prime}} j \omega \varepsilon_{o} \chi_{g}^{(0)}(\vec{n}) \stackrel{\leftrightarrow}{G}_{\mathrm{cav}}\left(\vec{n}, \vec{n}^{\prime}\right) \cdot \vec{E}\left(\vec{n}^{\prime}\right)=\frac{1}{\kappa} \vec{E}(\vec{n})
$$

The corresponding discretized Green's function was obtained from the continuous Green's function in Fourier-space by

$$
\begin{gathered}
\stackrel{\leftrightarrow}{G}_{\mathrm{cav}}\left(\vec{n}, \vec{n}^{\prime}\right)=\iint d k_{x} d k_{y} e^{j k_{x} d_{x}\left(n_{x}-n_{x}^{\prime}\right)} e^{j k_{y} d_{y}\left(n_{y}-n_{y}^{\prime}\right)} \\
\times \frac{4 \sin ^{2}\left(k_{x} d_{x} / 2\right) \sin ^{2}\left(k_{y} d_{y} / 2\right)}{\pi^{2} k_{x}^{2} k_{y}^{2} d_{x} d_{y}} \stackrel{\leftrightarrow}{G}_{\mathrm{cav}}\left(k_{x}, k_{y}\right)
\end{gathered}
$$

for these rectangular testing functions. For this work, a commercially available (IMSL) routine for generalized complex eigenvalue problems was used to solve Eq. (5).

Figure (2) shows the first three gain eigenvalues $\kappa(\omega)$ (two are essentially degenerate) plotted at

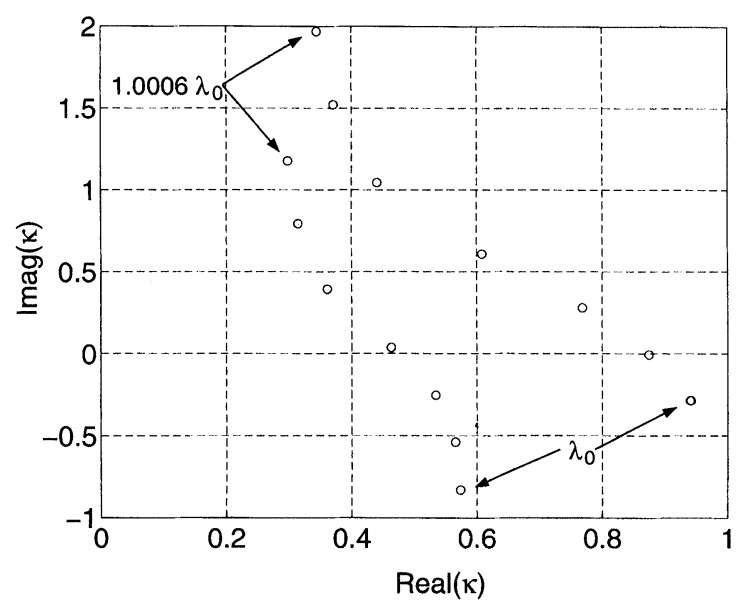

FIGURE 2 First three complex gain eigenvalues at discrete intervals in frequency; two are essentially degenerate. Lasing is possible only where the eigenvalue curves crosses the real axis, with the values of the gain eigenvalues at these crossings defining the threshold condition for lasing. 
constant intervals in frequency. Approximating the gain susceptibility available from the electronic system of the laser under bias as purely imaginary, lasing is only possible where one of the gain eigenvalue curves crosses the real axis, such that $\chi_{g}(\vec{r}, \omega)=\kappa(\omega) \chi_{g}^{(0)}(\vec{r})$ is also purely imaginary. The gain eigenvalue at the crossing frequency defines the threshold gain susceptibility. Approximating the gain susceptibility as purely imaginary is done here for illustrative purposes only. In reality the actual lasing modes of the system would be determined by the intersection of the gain eigenvalues with a curve describing the KramersKronig relation between the real and imaginary parts of the gain susceptibility. The large area active region considered in this example produces a very tight spacing of gain eigenvalues in frequency, but the gain amplitudes required for lasing are significantly different because of the different overlaps of the self-consistently calculated lasing modes with the active region. Figure (3) shows the field patterns of the first and third lasing modes inside the gain region.

\section{CONCLUSION}

The problem of obtaining the lasing modes and corresponding threshold conditions for VCSELs has been formulated as a frequency-dependent eigenvalue problem in required gain amplitudes and corresponding fields. Index and gain guiding are treated on an equal footing. The complex gain eigenvalues define necessary but not sufficient conditions for lasing. The actual lasing frequencies and modes that the VCSEL can support are then determined by matching the gain necessary for the optical system in both magnitude and phase to that available from the laser electronic system. With this formulation the lasing modes and corresponding threshold conditions are well defined even when the optical cavity boundaries and conventional cavity parameters such as the photon lifetime are not.
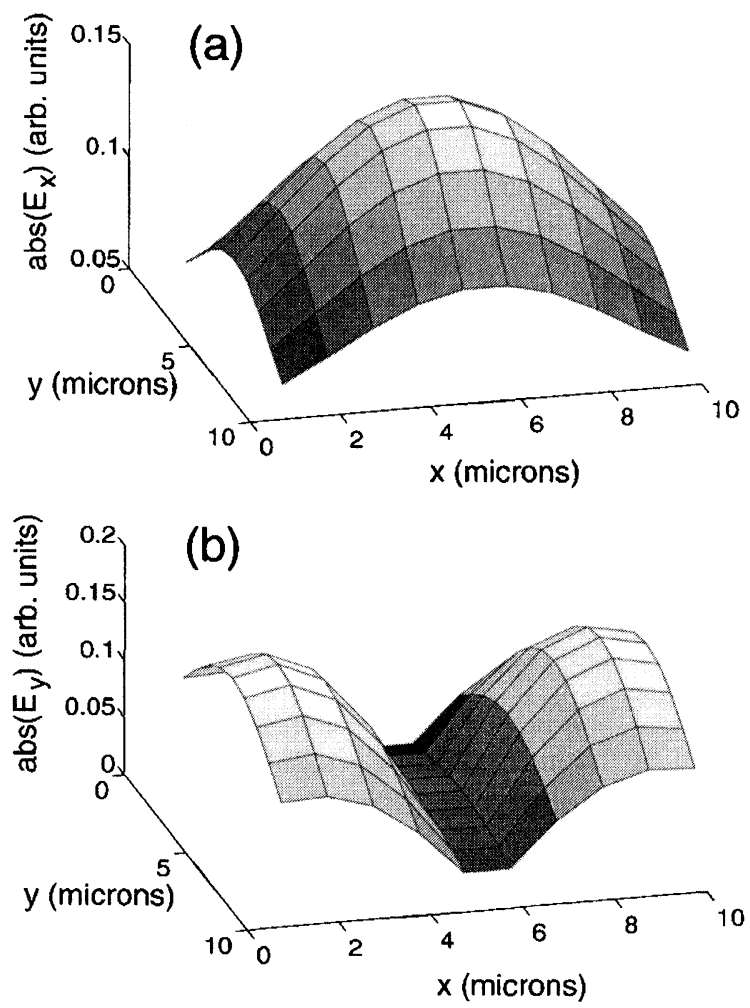

FIGURE 3 Lasing fields at (a) $\lambda \simeq 1.0003 \lambda_{o}$ and (b) $\lambda \simeq$ $1.0001 \lambda_{o}$ as a function of position within the gain region.

This formulation also has the practical advantage that the problem of obtaining the selfsupporting lasing fields of a VCSEL cavity is separated into two distinct and already thoroughly studied problems: that of finding the optical Green's functions for a fixed radiation source in an open cavity and that of solving a complex eigenvalue problem. Thus, this formulation provides a framework for the application of a large preexisting knowledge base to the relatively new challenge of modeling VCSELs. For example, in the specific implementation used for this work, the cavity Green's function was obtained by textbook methods [4-6] and the generalized complex eigenvalue problem was solved using a commercially available numerical routine. 


\section{Acknowledgements}

This material is based upon work supported by the Office of Naval Research.

\section{References}

[1] Grupen, and Hess, invited paper IWCE, Tempe Az, October 1995, to be published in VLSI Design.

[2] Lin, C. C. and Deppe, D. G. (1995). J. Lightwave Tech., 13,575 .

[3] Chow, W., Koch, S. and Sargent, M. III, Semiconductor Laser Physics (Springer-Verlag, Berlin, 1994) p. 55.

[4] Chew, W. C. Waves and Fields in Inhomogeneous Media (Van Nostrand Reinhold, New York, 1990) pp. 57-79, $375-418$.

[5] Chew, W. C. pp. 45-56.

[6] Born, M. and Wolf, E. Principles of Optics, Sixth Edition (corrected) (pergamon Press, Oxford, 1993) pp. 51-66.

[7] Balanis, C. A. Advanced Engineering Electromagnetics (John Wiley and Sons, New York, 1989) p. 692.

\section{Authors' Biographies}

Benjamin Klein received his B.S. and M.S. degrees in Electrical Engineering from the University of Wisconsin at Madison in 1994 and 1996, respectively. $\mathrm{He}$ is currently pursuing his Ph.D. in Electrical Engineering at the University of Illinois at Urbana-Champaign. His thesis research is the numerical simulation of vertical cavity surface emitting lasers.

Leonard F. Register is a Research Scientist in the Beckman Institute and Coordinated Science Laboratory at the University of Illinois at UrbanaChampaign. His current research interests include dissipative quantum transport, laser simulation, and leakage currents in thin oxides.

Karl Hess has dedicated the major portion of his research career to the understanding of electronic current flow in semiconductors and semiconductor devices with particular emphasis on effects pertinent to device miniaturization. His theories and use of large computer resources are aimed at complex problems with clear applications and relevance to miniaturization of electronics. $\mathrm{He}$ is currently the Swanlund Professor of Electrical and Computer Engineering, Professor of Physics, Adjunct Professor for Supercomputing Applications and a Research Professor in the Beckman Institute working on topics related to Molecular and Electronic Nanostructures. He has received numerous awards, for example the IEEE David Sarnoff Field Award for electronics in 1995.

Dennis G. Deppe is an Associate Professor in the Electrical and Computer Engineering Department at The University of Texas at Austin. He received the Ph.D. degree in 1988 from the University of Illinois at Champaign-Urbana, where he studied atomic diffusion in III-V semiconductor heterostructures, and semiconductor laser fabrication and characterization. After receiving the Ph.D. in 1988 he worked as a Member of Technical Staff at AT\&T Bell Laboratories in Murray Hill, New Jersey researching semiconductor lasers, and in 1990 accepted a position as Assistant Professor at The University of Texas at Austin. He was promoted to Associate Professor in 1994, and holds the Robert and Jane Mitchell Endowed Faculty Fellowship in Engineering. His research specialties include III-V semiconductor device fabrication, optoelectronics, and laser physics. He has published over 120 technical journal articles and holds 6 U.S. patents, and has received the National Science Foundation Presidential Young Investigator and Office of Naval Research Young Investigator Awards. 

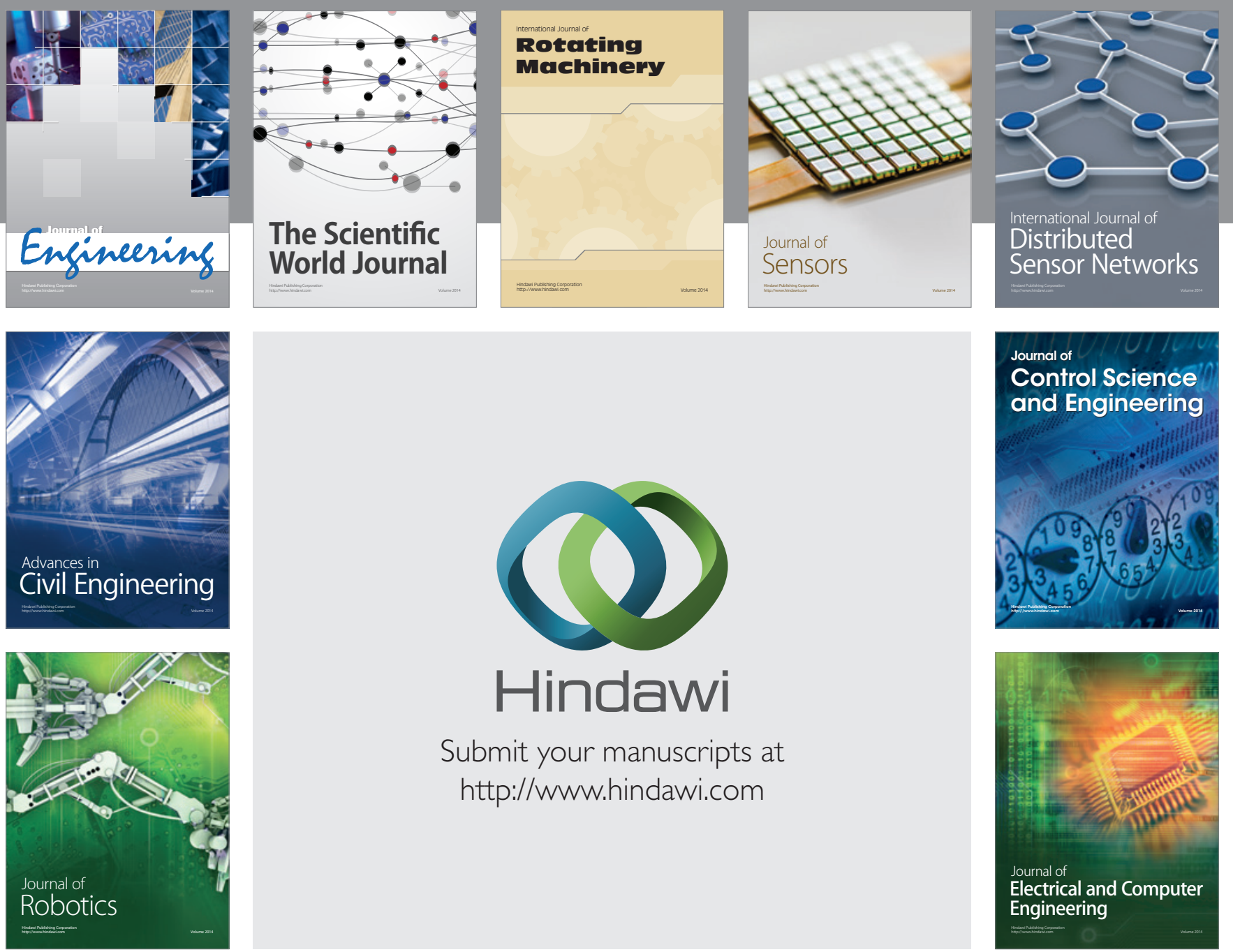

Submit your manuscripts at

http://www.hindawi.com
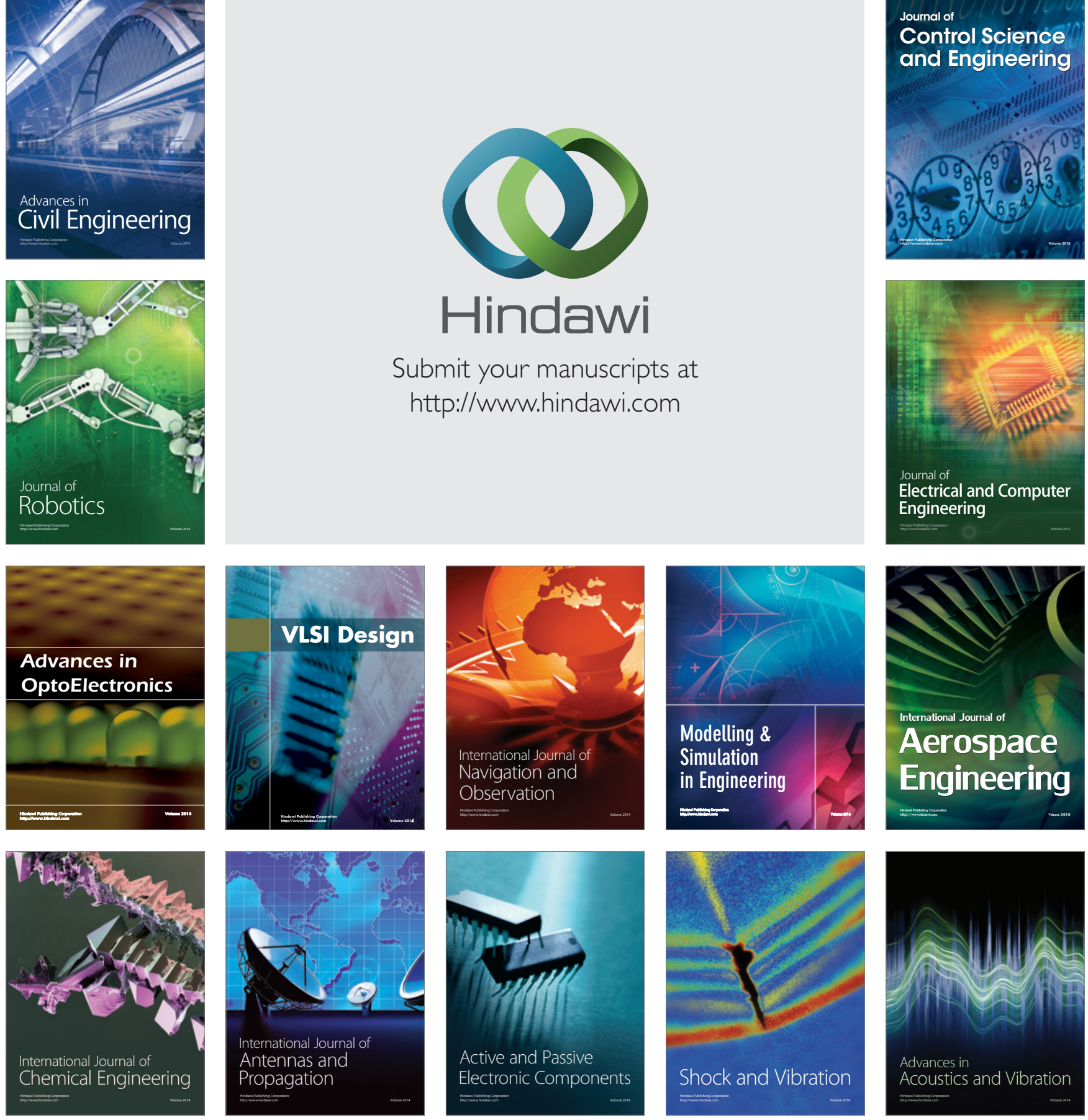